\title{
Innovative Gas-Cooled Reactors
}

\section{Jan Leen Kloosterman, ${ }^{1}$ Jim Kuijper, ${ }^{2}$ Colin Mitchell, ${ }^{3}$ and Guglielmo Lomonaco ${ }^{4}$}

\author{
${ }^{1}$ Delft University of Technology (TUD), 2628 Delft, The Netherlands \\ ${ }^{2}$ Nuclear Research \& Consultancy Group (NRG), 1755 ZG Petten, The Netherlands \\ ${ }^{3}$ AMEC-NNC, Knutsford WA16 8QZ, UK \\ ${ }^{4}$ Department of Mechanics, Nuclear and Production Engineering (DIMNP), \\ University of Pisa (UNIPI), 56126 Pisa, Italy
}

Correspondence should be addressed to Guglielmo Lomonaco, g.lomonaco@ing.unipi.it

Received 31 December 2009; Accepted 31 December 2009

Copyright (C) 2009 Jan Leen Kloosterman et al. This is an open access article distributed under the Creative Commons Attribution License, which permits unrestricted use, distribution, and reproduction in any medium, provided the original work is properly cited.

Innovative gas-cooled reactors, either with a thermal (HTGR) or fast (GCFR) neutron spectrum, are widely considered by academia, research centres, and industry to be among the most promising reactor concepts for the next generation. These reactors are characterized by a fully ceramic core and a noncorrosive coolant (helium or carbon dioxide), which is transparent for neutrons. Due to these characteristics, it is possible to have high operating temperatures.

$R \& D$ projects are currently under way in many countries from China, European Union, Japan, Russia, South Africa, South Korea, to the United States, of which many are cooperating through large international projects, such as the framework programmes of the EU, the Generation IV International Forum (GIF), and the International Project on Innovative Nuclear Reactors, and the Fuel Cycles (INPRO) programme of the IAEA.

In terms of safety and competitiveness, the high temperature gas-cooled reactor (HTGR) has very attractive characteristics. It is generally considered to be the most promising candidate at short term and seems to offer at least a partial solution to the growing world energy demand. The operational experience obtained from the HTTR and the HTR-10, in conjunction with the experience already available from other reactors and research projects, constitutes a strong technological basis for the commercial development of HTGRs. Additionally, the fuel cycle flexibility of the HTGR offers the possibility to use this reactor type for the reduction of plutonium and minor actinides stockpiles, as recent studies indicate.

Recently, the gas cooled fast reactor (GCFR) has also gained the interest of industry and the international scientific community. Due to the positive characteristics common to all fast reactors (the improved sustainability by generation of fuel from fertile nuclides and the possibility for efficient burning of nuclear waste) combined with the advantages of gas coolants (no phase change, and no nuclear or chemical reactions in the core), the GCFRs represent a very interesting prospect for future nuclear technology. Their development takes advantage of the high temperature gas coolant technology arising from the HTGRs.

In view of the above, it has been decided to bring out the special issue "Innovative gas-cooled reactors". Looking to the manuscripts, they could be subdivided into three groups.

(1) Three papers are related to HTR/VHTR systems (mainly safety and fuel cycles related aspects).

(2) Two papers are related to symbiotic fuel cycles and scenarios involving LWR, HTR and GCFR.

(3) Six papers are related to GCFR systems (covering many aspects, from the historical background to the present international research activities).

As a result, this widespread interest in innovative gascooled reactors from industry to research centres and universities is a stimulus for the rejuvenation of the industry, attracting young talents to the universities to become the scientists and engineers of the future.

Jan Leen Kloosterman Jim Kuijper Colin Mitchell

Guglielmo Lomonaco 

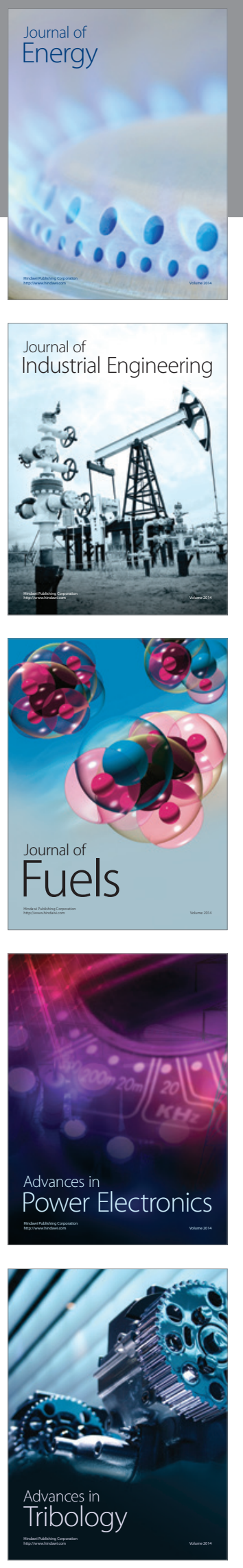
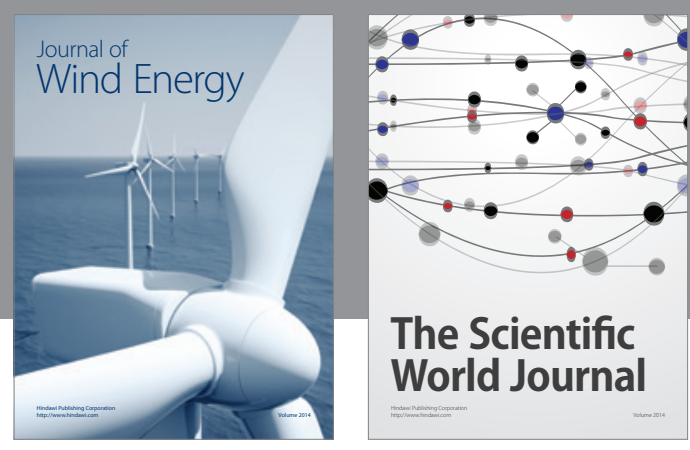

The Scientific World Journal

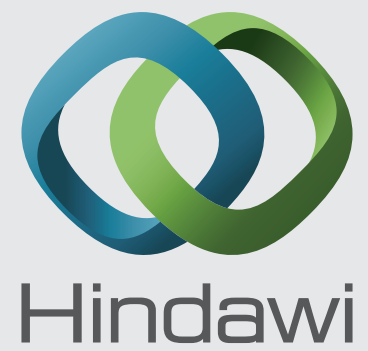

Submit your manuscripts at http://www.hindawi.com
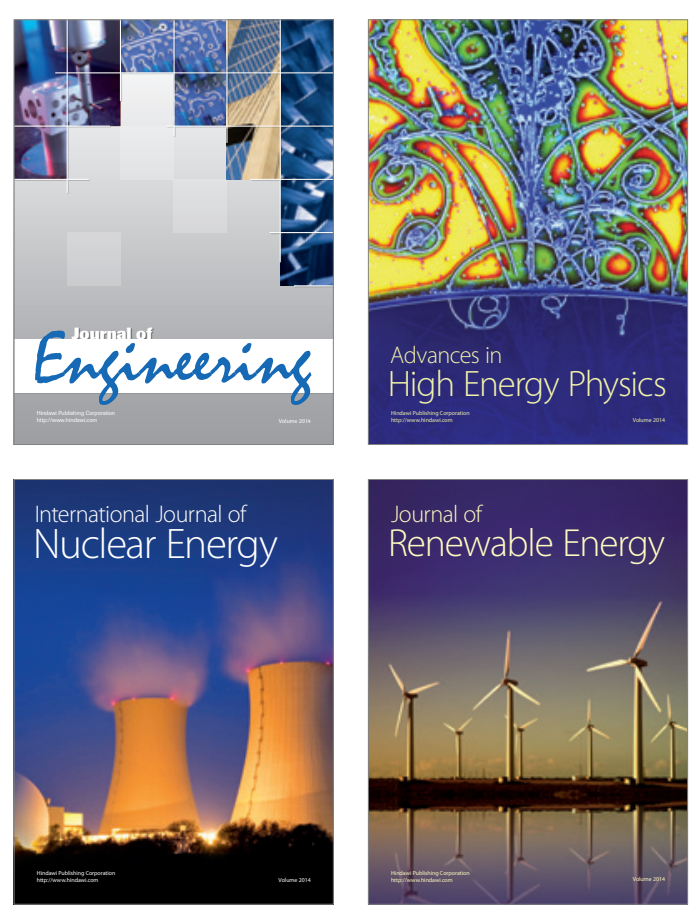

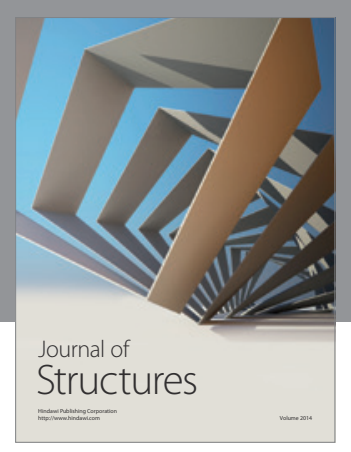

Rotating
Mechinery
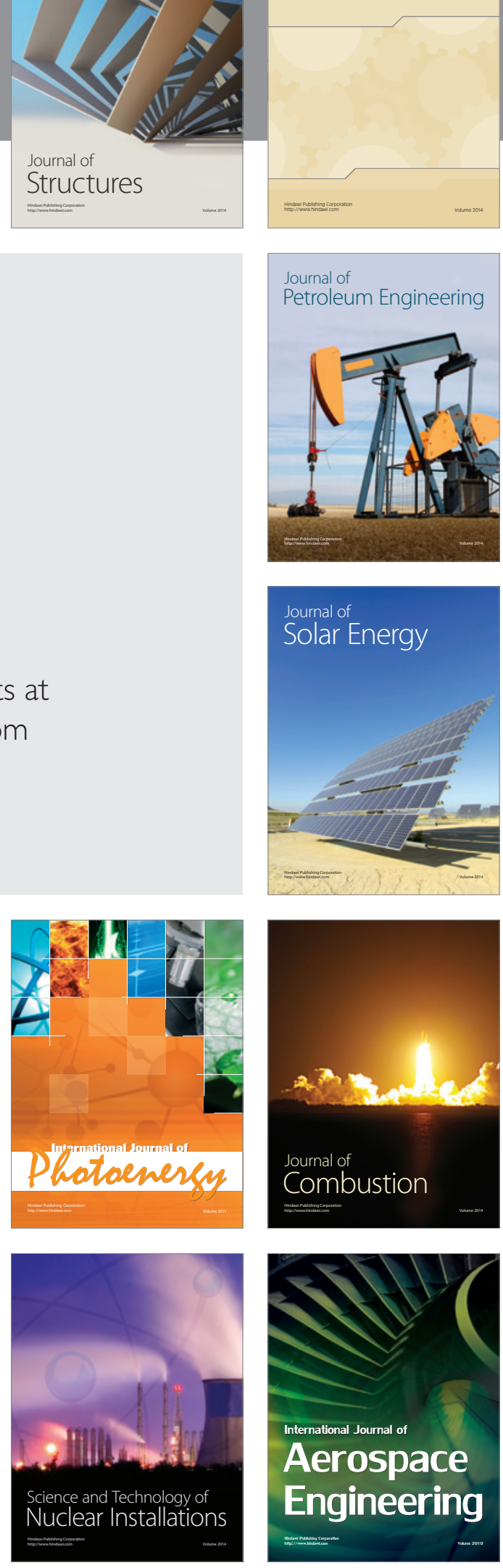